\title{
BreastCore
}

\section{Proton Therapy for Primary Breast Cancer}

\author{
Eugen B. Hug \\ MedAustron Ion Therapy Center, Wiener Neustadt, Austria
}

\section{Keywords \\ Breast cancer - Cardiotoxicity · Proton therapy}

\section{Summary}

Proton therapy reduces the integral dose received by normal tissues due to its physical properties of dose deposition in the Bragg peak. In a small but significant percentage of patients requiring adjuvant radiotherapy (RT) for left-sided breast cancer, photon-based RT can lead to cardiac complications during long-term followup. The risk of cardiac complications is correlated with the dose to the coronary arteries and to the general 'mean heart dose'. Dosimetric comparison analysis has identified advantages of proton therapy in accomplishing sparing of the heart with increasing target complexity while permitting uncompromised target coverage of the chest wall \pm breast plus draining lymphatics. Early clinical data indicate good clinical tolerance to proton therapy without unexpected complications. Several clinical trials are presently ongoing to prospectively confirm a clinical benefit and to identify the subgroup of patients benefitting most from proton therapy for breast cancer.

(c) 2018 S. Karger GmbH, Freiburg

\section{Introduction}

Proton therapy has been in routine clinical use since the mid1980s with the first hospital-based proton therapy center becoming operational at Loma Linda, CA, USA, in 1991. The attractiveness of proton therapy and its potential advantages over photon therapy are based primarily on its physical dose distribution. In an energydependent manner, proton therapy will deposit the majority of its energy in tissue depths defined by the Bragg peak. In practice, this translates into i) a reduced entrance dose, ii) the ability to deliver the peak energy to target volumes of irregular 3-dimensional (3D) shape using pencil-beam scanning technology, and iii) a sharp dose falloff following deposition of energy in the target volume. Within several millimeters, the exit dose literally drops off from 90 to $10 \%$, resulting in the virtual absence of an exit dose. Innumerable dose comparisons have confirmed that compared to most modern photon technologies like stereotactic body radiation therapy (SBRT), volumetric arch therapy (VMAT), and intensity modulated radiotherapy (IMRT) proton therapy results in a decreased integral dose to normal tissues exposed to unnecessary radiation. Initial clinical indications focused on relatively rare tumors such as skull base tumors, uveal melanomas, and sarcomas, followed by its application in pediatric tumors. In the last $10-15$ years, proton therapy has become more readily available in major cancer centers. With this more widespread introduction, epidemiologically frequent tumors have been explored including head and neck malignancies, gastrointestinal and thoracic malignancies, and breast cancer.

Worldwide, more than 140,000 patients have been treated with proton therapy (www.ptcog.ch). Although there is still a general paucity of data based on randomized clinical trials, its principle effectiveness, safety, and feasibility are no longer questioned. More than 100 prospective clinical trials are presently recruiting patients [1]. However, proton therapy is still an emerging technology (also in the field of breast cancer) that requires prospective data to identify the most suitable subgroup of patients within a specific disease entity for whom proton therapy will be of most value and result in a clinically significant difference over the use of photons.

\section{Rationale for the Use of Proton Therapy in Breast Cancer}

Conventional radiation therapy (RT) can potentially result in an increased risk of heart disease in women with left-sided breast cancer when complex irradiation of the chest wall and lymphatics is required. In 2006, Harris et al. [2], for the group at the University of Pennsylvania, reported on a review of 961 patients treated between 1977 and 1994 for breast cancer. At a median follow-up of 12 years and with equal distribution of risk factors, patients that received RT for left-sided breast cancer compared to those under-

\section{KARGER}

() 2018 S. Karger GmbH, Freiburg

Fax +497614520714 
Proton Therapy - whole breast with boost following lumpectomy

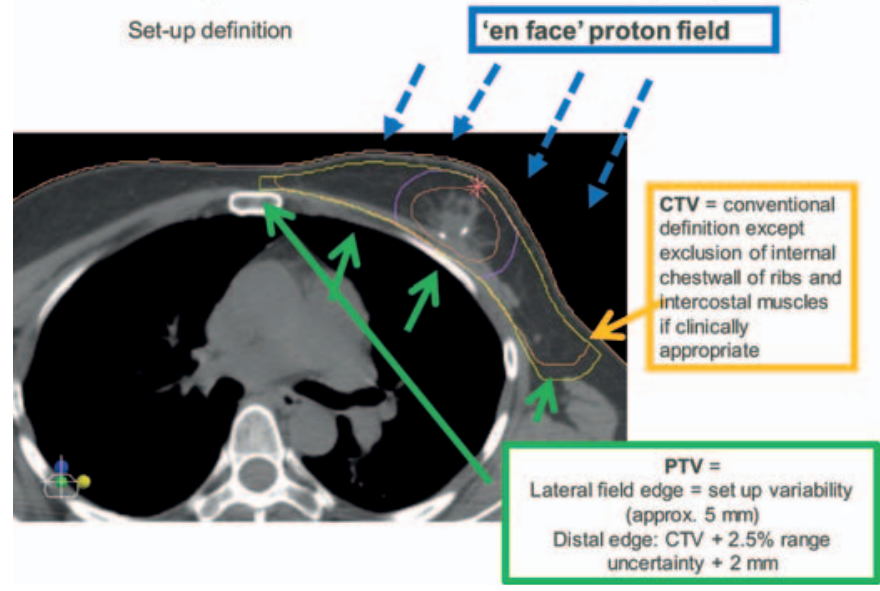

Fig. 1. Schematic outline of planning criteria for adjuvant postoperative proton therapy following lumpectomy for breast cancer targeting the breast/chest wall (as shown) and draining lymphatics (not shown).

going RT for right-sided cancer were noted to have an increased risk of coronary artery disease $(\mathrm{p}<0.001)$. At 20 years after RT, the actuarial freedom-from coronary artery disease rates were $90 \%$ for right-sided and $75 \%$ for left-sided breast cancer patients. Rates started to separate at approximately 10 years of follow-up. Coronary artery disease translated into a significant risk of myocardial infarction. In 2013, this work resulted in a publication in the New England Journal of Medicine [3]: The 'mean heart dose' was identified as a prognostic indicator, correlating directly with incidence rates for coronary artery events. A 7.4\% increase was noted per Gray mean heart dose without a minimum threshold dose. Previously, the anatomic location of coronary artery stenosis had been correlated with isodoses of RT.

Radiation is well known to potentially cause damage to any part of the heart either acutely or as a late effect including but not limited to coronary artery disease, acute myocardial infarction, acute heart failure due to cardiomyopathy or effects on the conducting system, late valve stenosis via calcification, and also acute pericardial effusion. In patients with breast cancer, coronary artery disease appears to be the most frequently observed cause [4].

\section{Technical Aspects}

Proton RT to the chest wall and draining lymphatics is typically delivered by matching several fields [5]. In the majority of patients, the chest wall \pm breast is treated using a direct en-face field. $4 \mathrm{D}$ computed tomography (CT) scans typically reveal chest wall motion primarily affecting the air gap, i.e., the chest moving towards or away from the beam direction rather than in a superior to inferior or lateral motion [5]. Hence, although the chest wall is in principle a motion-dependent anatomic site, motion mitigation is by and large not required since the effects of small air gap changes have a minimal influence on the resulting dose distribution [5]. Using a direct en-face approach, typically about 5-10 $\mathrm{mm}$ of the
Fig. 2. Isodose

distribution of a proton plan for stage III breast cancer.

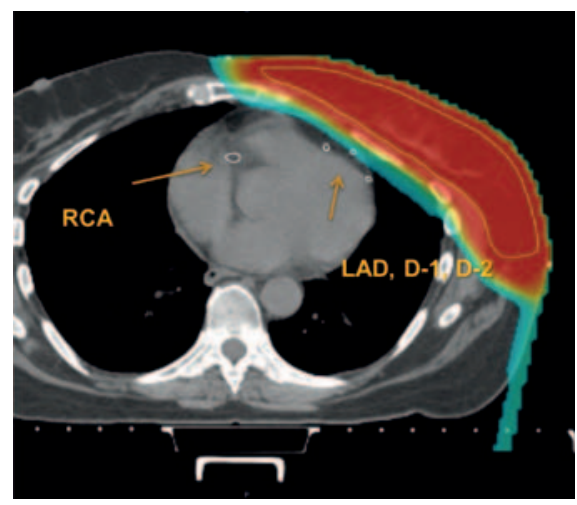

innermost chest wall are excluded from the direct target volume and are used as soft tissue and bone density to facilitate the stopping power of protons. The innermost $5-10 \mathrm{~mm}$ of chest wall accomplish a dose reduction to approximately $20 \%$ of the prescription dose. The resulting doses overshoot into involved lung, and effects on the heart are subsequently modest.

Such an approach requires a careful robustness analysis based on $4 \mathrm{D}$ planning $\mathrm{CT}$ to judge motion uncertainty and to include factors like range uncertainty as schematically shown in figure 1 . Figure 2 represents a typical isodose distribution of a left-sided breast cancer requiring inclusion of the chest wall and the axillary, supraclavicular, and internal mammary lymph nodes (LN). Of note is the sharp dose falloff towards the heart.

A recent planning comparison by Patel et al. [6] for the $\mathrm{MGH}$ Group did not detect significant differences in cardiac sparing potential by use of deep inspiration breath hold compared to free breathing or for passively scattered proton delivery versus active scanning.

\section{Preclinical Dosimetric Studies}

In 2010, an initial dosimetric treatment planning comparison using pencil-beam scanning was published by Ares et al. [7] for the Paul Scherrer Institute. Comparing photon with proton techniques, the authors documented an increasing dosimetric advantage for protons with increasing target complexity, i.e., from chest wall irradiation to chest wall plus axillary LN to chest wall plus axillary, supraclavicular, and internal mammary LN.

Fontanilla et al. [8] raised the issue that standard photon techniques frequently require a compromise to be made between prescribed dose target coverage and organ-at-risk (OAR) dose constraints. The percentage of RTOG (Radiation Therapy Oncology Group) guideline-based contours covered by $90 \%$ of the 50 Gy prescribed was only $74 \%$ of the chest wall, $84 \%$ of level $1 \mathrm{LN}, 88 \%$ of level $2 \mathrm{LN}$, 93\% of level $3 \mathrm{LN}, 84 \%$ of supraclavicular LN, and $80 \%$ of internal mammary LN, and improving the target coverage would result in higher OAR doses. However, such a compromise would not be required with the use of protons as documented in the publication by Ares et al. [7] amongst others. 
Fagundes et al. [5] compared protons with photon-based 3D conformal RT, helical tomotherapy, and VMAT in 10 patients with stage III left-sided breast cancer. The mean heart dose was significantly $(\mathrm{p}<0.001)$ decreased with the use of protons $(1.2 \pm 0.42$ Gy(relative biological effectiveness (RBE)) compared with 3D conformal RT $(6.8 \pm 2.08 \mathrm{~Gy})$, helical tomotherapy (10.2 $\pm 1.6 \mathrm{~Gy})$, and VMAT $(8.2 \pm 1.13 \mathrm{~Gy})$. The heart-sparing benefit of protons persisted even for proton plans with inclusion of internal mammary LN compared to photon plans without inclusion of internal mammary LN.

Stick et al. [9] in a treatment planning comparison study addressed both, a joint estimation of the risk of recurrence caused by inadequate radiation dose coverage of $\mathrm{LN}$ targets and the risk of cardiac toxicity caused by radiation exposure to the heart. 'Delivered' photon plans were compared with 'realistic' proton plans in $41 \mathrm{pa}-$ tients requiring adjuvant comprehensive nodal irradiation for leftsided breast cancer. Cardiotoxicity risk was estimated based on the model by Darby et al. [3], and risk of recurrence following a compromise in LN coverage was estimated with a linear dose-response model fitted to the recurrence data from the EORTC (European Organisation for Research and Treatment of Cancer) 22922/10925 and NCIC-CTG (National Cancer Institute of Canada Clinical Trials Group) MA.20 randomized controlled trials. The excess absolute risk of cardiac morbidity was small for photon therapy with median values of 1 and $0.5 \%$ with and without cardiac risk factors, respectively, but even lower with proton therapy $(0.13$ and $0.06 \%$, respectively). The median estimated excess absolute risk of breast cancer recurrence after 10 years was $0.1 \%$ with photons and $0.02 \%$ with protons. The authors concluded that modern photon therapy yields a limited risk of cardiac toxicity in most patients, but proton therapy can reduce the predicted risk of cardiac toxicity by up to $2.9 \%$ and the risk of breast cancer recurrence by $0.9 \%$ in individual patients.

The dosimetric advantages of protons versus modern photon techniques were confirmed by several other publications (Cuaron et al. [10], Mast et al. [11], and Bradley et al. [12]). The photon versus proton planning comparison study by MacDonald et al. [13] revealed for comprehensive treatment with inclusion of internal mammary LN an ipsilateral lung volume receiving $20 \mathrm{~Gy}$ (V-20) of $30 \%$ with photons versus $15 \%$ with protons, and a mean heart dose of 4-10 Gy compared to <1 Gy, respectively.

\section{Clinical Indications, Early Outcome Data, and Status of Clinical Studies}

In general, proton therapy approaches for breast cancer can be grouped into partial breast irradiation for early-stage breast cancer and complex RT for locally advanced breast cancer with inclusion of the lymphatics.

\section{Accelerated Partial Breast Irradiation}

Bush et al. [14] for the Loma Linda Group reported in 2014 on 100 patients treated for invasive non-lobular carcinoma with a maximal tumor dimension of $3 \mathrm{~cm}$, having undergone partial mas- tectomy with negative margins and negative axillary LN sampling. Postoperative proton RT was delivered as $4 \mathrm{~Gy}(\mathrm{RBE})$ in 10 fractions, 1 treatment daily over 2 weeks. Multiple fields were treated daily, and skin-sparing techniques were used. At a median followup of 60 months, ipsilateral recurrence-free survival was 97\%, no cases of grade 3 or higher acute skin reaction were noted, and late skin reactions included 7 cases of grade 1 telangiectasia. These encouraging data resulted in a prospective clinical trial (BRE007-12) initiated by the Proton Therapy Cooperative Group (PTCOG) which has completed accrual and is presently in its follow-up phase.

Early data indicate that proton therapy for partial breast irradiation is non-inferior to modern photon techniques. However, in light of the overall small target volumes and the excellent capability of photon therapy to obtain target volume coverage, it remains to be determined whether there is a distinct long-term advantage for proton therapy.

\section{Proton Therapy for Locoregionally Advanced Breast Cancer or} for Patient with an Unfavorable Anatomy Requiring Postop RT to the Chest Wall/Breast $\pm L N$

The rationale for the use of proton therapy is based on the goals of i) providing maximum sparing of the heart and coronary arteries and thus reducing the incidence of severe cardiac morbidity and cardiac death, ii) reducing the integral volume of normal tissue exposure to RT and thus reducing the incidence of second malignancies well established in prospective clinical photon trials (primarily late induction of contralateral breast cancer and non-small cell lung cancer), iii) providing optimum dose coverage of target volumes without compromise and thus potentially improving local control, and iv) improving quality of life.

Several institutions have reported early outcome data, notably the groups at Massachusetts General Hospital (MacDonald et al. [13]), the Procure Proton Therapy Center in New Jersey (Cuaron et al. [10]), and the Florida Proton Therapy Institute (Bradley et al. [12]). These first publications unanimously reported good tolerance with no increased skin or cosmetic toxicities using standard fractionation schedules at generally accepted total dose levels. They confirmed in clinical practice that indeed a mean heart dose of 2 $\mathrm{Gy}(\mathrm{RBE})$ is rarely exceeded. Skin toxicities included pronounced grade 2 or early grade 3 transient skin toxicities (Common Terminology Criteria for Adverse Events (CTCAE) vs. 4.0) but were considered overall acceptable. MacDonald et al. [13] reported acute toxicities in 12 women treated with postmastectomy proton radiation with or without reconstruction to a dose of $50.4 \mathrm{~Gy}(\mathrm{RBE})$ to the chest wall and 45-50.4 Gy(RBE) to the regional lymphatics. In this prospective clinical study, the maximum skin toxicity during radiation was grade 2 (CTCAE). Maximum fatigue was CTCAE grade 3. Verma et al. [15] reported on 91 patients treated with adjuvant proton beam therapy (PBT) targeting the intact breast/chest wall and comprehensive regional nodes including the axilla, supraclavicular fossa, and internal mammary LN. The median PBT dose was $50.4 \mathrm{~Gy}(\mathrm{RBE})$ with a subsequent boost as clinically indicated $(\mathrm{n}=61$, median $10 \mathrm{~Gy}(\mathrm{RBE})$ ). At a median follow-up of 15.5 
months, grade 1,2 , and 3 dermatitis had occurred in 23, 72, and $5 \%$, respectively. The median time to resolution of dermatitis was 32 days after completion of RT.

Early publications provide data about the safety and feasibility of proton therapy and acute toxicities. In a systematic review of published clinical data, Kammerer et al. [16] concluded that proton therapy often decreases the mean heart dose by a factor of 2 or 3 , i.e., $1 \mathrm{~Gy}$ with proton therapy versus $3 \mathrm{~Gy}$ with conventional 3D conformal RT and 6 Gy for IMRT. It is yet premature to expect long-term data about any reduction in cardiac morbidity or mortality, cosmetic outcome, or second malignancies.

In 2013, the author started a prospective phase II study of postoperative cardiac-sparing proton RT for patients with a stage II-III locoregional, non-metastatic breast cancer requiring whole breast or chest wall irradiation with LN irradiation. This study is multiinstitutionally conducted by the Proton Therapy Collaborative Group (PCG, trial no. BRE008-12). At present it has accrued $>150$ patients.

A long-term study is currently accruing patients in the USA funded by the Patient-Centered Outcomes Research Institute (PCORI), a nonprofit, non-governmental organization authorized by the Patient Protection Affordable Care Act. For the purpose of conducting a pragmatic randomized trial of proton versus photon therapy for patients with stage II-III breast cancer, the Radiotherapy Comparative Effectiveness Consortium was founded which includes 22 institutions. Patients are randomized to photons versus protons, but treatment will be conducted according to the institutional practice (the paradigm of 'pragmatic trials'). Primary endpoints are major cardiovascular event (MCE) reduction by protons (hypothesizing a reduction in the 10 -year MCE rate from 6.3 to $3.8 \%$ compared to photons). In parallel, primary patient-reported health-related quality of life (HRQOL) outcome data will be collected.

One of the challenges in assessing the long-term beneficial effects of protons is the long observation period required. Efforts to identify prognostic factors that will permit an early estimate of the incidence or severity of cardiac damage have been published, but no single factor has thus far proven to be a reliable predictor [17]. This trial is notable for a major research component examining the association of radiation distribution with MCE and HRQOL in an effort to develop predictive models. Recent research has correlated various pathophysiologic events with the expression of certain biomarkers; examples are the correlation of inflammation with GDF15, microvascular dysfunction with PIGF, oxidative stress with $\mathrm{MPO}$, and myocyte injury with TnT. Hence, biomarkers will be studied to assess if an early increase can predict changes according to RT and if a more adverse biomarker profile for photon therapy can be identified. In addition, functional alterations in ventricular structure, systolic function, etc. will be evaluated using echocardiography. All these efforts are aimed at developing a risk prediction algorithm using biomarkers, echocardiographic ultrasound data, and clinical variables to identify the patient at increased risk of radiation-induced cardiotoxicity. This trial opened in 2016 and aims to recruit 1,750 patients.

\section{Identification of Disease-Specific Patient Subgroups Most} Suitable for Proton Therapy

Thus far, all available publications indicate that even for the most complex irradiation of the chest wall including the lymphatics proton therapy can consistently and routinely keep the mean cardiac dose at or below $3 \mathrm{~Gy}(\mathrm{RBE})$ based on standard dose regimens. In contrast, photon RT may or may not be able to keep radiation doses in these patients at equally low levels. Various institutions use varying techniques to optimize dose distribution, and depending on the individual anatomy of the patient, i.e. chest wall curvature and proximity of the heart to the chest wall, the resulting dose to the heart and coronaries can vary significantly. Although in principle the mean heart dose is likely to increase with increasing complexity and thus stage of disease, individual results may vary. In other words, in some patients even a highly complex photon radiotherapy plan might be able to accomplish reasonable limitation of the mean cardiac dose, whereas in certain patients with an unfavorable anatomy, a photon plan might result in an unacceptably high mean heart dose even for stage II disease. It is one of the goals of current clinical trials to prospectively gather data and establish a treatment modality algorithm and to identify the subgroup of patients that will benefit most from proton RT.

At present, several factors are predictive of an increased MCE risk following breast irradiation for left-sided breast cancer: A radiation treatment plan resulting in a significantly elevated mean heart dose AND i) life expectancy > 15-20 years), ii) pre-existing cardiac disease or cardiac comorbidity, and iii) concurrent use of cardiotoxic systemic therapy (e.g., adriamycin or herceptin). Already today, if one or more of these factors apply, the radiation oncologist may be inclined to discuss with the patient a potential referral to a proton center.

\section{Conclusion}

Proton therapy is rapidly evolving as a radiation tool for women with left-sided breast cancer that require comprehensive irradiation. Proton therapy can limit unnecessary doses delivered to the heart and thereby is expected to reduce the risk of long-term cardiac morbidity. Identifying prognostic factors and the subgroup of patients in whom this benefit will be most significant is the subject of currently ongoing perspective clinical trials.

\section{Disclosure Statement}

The author does not have any conflicts of interest to declare. 


\section{References}

1 Mishra MV, Aggarwal S, Bentzen SM, Knight N, Mehta MP, Regine WF: Establishing evidence-based indications for proton therapy: an overview of current clinical trials. Int J Radiat Oncol Biol Phys 2017;97: 228-235.

2 Harris EE, Correa C, Hwang WT, Liao J, Litt HI, Ferrari VA, Solin LJ: Late cardiac mortality and morbidity in early-stage breast cancer patients after breast-conservation treatment. J Clin Oncol. 2006;24:4100-4106.

3 Darby SC, Ewertz M, McGale P, Bennet AM, BlomGoldman U, Brønnum D, Correa C, Cutter D, Gagliardi G, Gigante B, Jensen MB, Nisbet A, Peto R, Rahimi K, Taylor C, Hall P: Risk of ischemic heart disease in women after radiotherapy for breast cancer. N Engl J Med 2013;368:987-998.

4 Nilsson G, Holmberg L, Garmo H, Duvernoy O, Sjögren I, Lagerqvist B, Blomqvist C: Distribution of coronary artery stenosis after radiation for breast cancer. J Clin Oncol 2012;30:380-386.

5 Fagundes M, Hug EB, Pankuch M, Fang C, McNeeley S, Mao L, Lavilla M, Schmidt S, Ward C, Cahlon O, Hartsell W: Proton therapy for local-regionally advanced breast cancer maximizes cardiac sparing. Int J Particle Ther 2015;1:827-844.

6 Patel SA, Lu HM, Nyamwanda JA, Jimenez RB, Taghian AG, MacDonald SM, Depauw N: Postmastectomy radiation therapy technique and cardiopulmonary sparing: a dosimetric comparative analysis between photons and protons with free breathing versus deep inspiration breath hold. Pract Radiat Oncol 2017; 7:e377-e384.
7 Ares C, Khan S, Macartain AM, Heuberger J, Goitein G, Gruber G, Lutters G, Hug EB, Bodis S, Lomax AJ: Postoperative proton radiotherapy for localized and locoregional breast cancer: potential for clinically relevant improvements? Int J Radiat Oncol Biol Phys 2010; 76:685-697.

8 Fontanilla HP, Woodward WA, Lindberg ME, Kanke JE, Arora G, Durbin RR, Yu TK, Zhang L, Sharp HJ, Strom EA, Salehpour M, White J, Buchholz TA, Dong L: Current clinical coverage of Radiation Therapy Oncology Group-defined target volumes for postmastectomy radiation therapy. Pract Radiat Oncol 2012;2: 201-209.

$\rightarrow$ Stick LB, Yu J, Maraldo MV, Aznar MC, Pedersen AN, Bentzen SM, Vogelius IR: Joint estimation of cardiac toxicity and recurrence risks after comprehensive nodal photon versus proton therapy for breast cancer. Int J Radiat Oncol Biol Phys 2017;97:754-761.

10 Cuaron JJ, Chon B, Tsai H, Goenka A, DeBlois D, Ho A, Powell S, Hug E, Cahlon O: Early toxicity in patients treated with postoperative proton therapy for locally advanced breast cancer. Int J Radiat Oncol Biol Phys 2015;92:284-291.

11 Mast ME, Vredeveld EJ, Credoe HM, van Egmond J, Heijenbrok MW, Hug EB, Kalk P, van KempenHarteveld LM, Korevaar EW, van der Laan HP, Langendijk JA, Rozema HJ, Petoukhova AL, Schippers JM, Struikmans H, Maduro JH: Whole breast proton irradiation for maximal reduction of heart dose in breast cancer patients. Breast Cancer Res Treat 2014;148:3339.
2 Bradley JA, Mendenhall NP: Novel radiotherapy techniques for breast cancer. Annu Rev Med 2018;69:277288.

13 MacDonald SM, Patel SA, Hickey S, Specht M, Isakoff SJ, Gadd M, Smith BL, Yeap BY, Adams J, Delaney TF, Kooy H, Lu HM, Taghian AG: Proton therapy for breast cancer after mastectomy: early outcomes of a prospective clinical trial. Int J Radiat Oncol Biol Phys 2013;86:484-490.

14 Bush DA, Do S, Lum S, Garberoglio C, Mirshahidi H, Patyal B, Grove R, Slater JD: Partial breast radiation therapy with proton beam: 5-year results with cosmetic outcomes. Int J Radiat Oncol Biol Phys 2014;90:501505.

15 Verma V, Iftekaruddin Z, Badar N, Hartsell W, HanChih Chang J, Gondi V, Pankuch M, Gao M, Schmidt S, Kaplan D, McGee L: Proton beam radiotherapy as part of comprehensive regional nodal irradiation for locally advanced breast cancer. Radiother Oncol 2017; 123:294-298.

16 Kammerer E, Guevelou JL, Chaikh A, Danhier S, Geffrelot J, Levy C, Saloux E, Habrand JL, Thariat J: Proton therapy for locally advanced breast cancer: a systematic review of the literature. Cancer Treat Rev 2018; 63:19-27.

17 Serrano NA, Mikkelsen R, Canada J, Mezzaroma E, Weiss E, Abbate A: Biomarkers of cardiac injury in patients undergoing thoracic radiation therapy. Int J Cardiol 2016;223:507-509. 